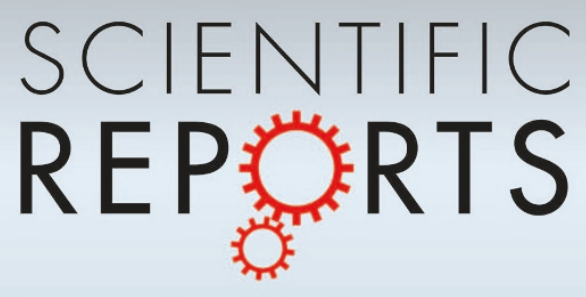

OPEN

SUBJECT AREAS:

APPLIED PHYSICS

PHYSICS

ELECTRICAL AND ELECTRONIC ENGINEERING

DESIGN, SYNTHESIS AND PROCESSING

Received

27 November 2012

Accepted

15 March 2013

Published

3 April 2013

Correspondence and requests for materials should be addressed to X.L. (luxinpei@hotmail.

com)

\title{
From short pulses to short breaks: exotic plasma bullets via residual electron
} control

\author{
YuBin Xian ', Peng Zhang ${ }^{2}$, XinPei Lu' , XueKai Pei' , ShuQun Wu' , Qing Xiong' \& Kostya (Ken) Ostrikov $3,4,1$
}

\begin{abstract}
'State Key Laboratory of Advanced Electromagnetic Engineering and Technology, Huazhong University of Science and Technology, Wuhan, Hubei 430074, People's Republic of China, ${ }^{2}$ Institute 601, Shenyang, Liaoning, 1 10035, People's Republic of China, ${ }^{3}$ CSIRO Materials Science and Engineering, PO Box 218, Lindfield NSW 2070, Australia, ${ }^{4}$ School of Physics, The University of Sydney, Sydney NSW 2006, Australia.
\end{abstract}

Plasma plumes with exotically segmented channel structure and plasma bullet propagation are produced in atmospheric plasma jets. This is achieved by tailoring interruptions of a continuous DC power supply over the time scales of lifetimes of residual electrons produced by the preceding discharge phase. These phenomena are explained by studying the plasma dynamics using nanosecond-precision imaging. One of the plumes is produced using $2-10 \mu$ s interruptions in the $8 \mathrm{kV}$ DC voltage and features a still bright channel from which a propagating bullet detaches. A shorter interruption of $900 \mathrm{~ns}$ produces a plume with the additional long conducting dark channel between the jet nozzle and the bright area. The bullet size, formation dynamics, and propagation speed and distance can be effectively controlled. This may lead to micrometer- and nanosecond-precision delivery of quantized plasma bits, warranted for next-generation health, materials, and device technologies.

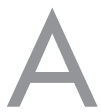

tmospheric-pressure non-equilibrium plasmas have recently received a major attention due to their widespread applications in surface and materials processing, biomedical engineering, and medicine $\mathrm{e}^{1-5}$. Among them, plasma jets have been of particular interest owing to the highly-focused treatment spots, portability, near-room temperature of the plasma plume, as well as demonstrated efficacy and flexibility in a broad range of applications spanning from cancer treatment to nanotechnology ${ }^{6-11}$. The plasma jets feature a very unique dynamic behaviour compared to other types of atmospheric-pressure plasmas. Indeed, the discovery of fast-propagating plasma bullets (also termed pulsed atmospheric pressure streamers (PAPSs)) opens an exciting possibility to control small bits of plasmas over sub-microsecond and possibly even nanosecond time scales ${ }^{12-16}$.

However, this fascinating phenomenon is still challenging to control and is far from being understood despite of a few mechanisms proposed ${ }^{17-24}$. Since these discharges are generated by time-varying electric fields, a series of breakdowns produces residual electrons within the channel of the plasma jet. The reported density of the residual electrons is vastly different by many orders of magnitude (e.g., from $\sim 10^{2}$ to $\left.\sim 10^{2} \mathrm{~cm}^{-3}\right)^{17,24-30}$. Despite this quantitative uncertainty, it is generally believed that the residual electron dynamics plays a major role in the formation of the still plasma channel and the propagating plasma bullets that detach from it ${ }^{19}$. However, it is extremely difficult to control and monitor such a dynamic behaviour, mostly because of the very high (e.g., nanosecond) precision required as well as large uncertainties in the residual electron lifetimes. When using conventional photography, the bullet-prone plasma plumes commonly appear as dim glow channels. This is why despite the excellent recent progress in the studies of the plasma streamer phenomena, the understanding of the mechanisms of the effect of the residual electrons on the streamer propagation is far from being complete.

To control the dynamics of the residual electrons, and hence, the plasma channel structure and the plasma bullet propagation, we customized the DC voltage to merely vary it over the timescales comparable with the expected lifetime of the residual electrons. In this way, by retaining the residual electrons long enough to affect the breakdown dynamics, here we report on the two exotic bullet-prone plume structures both featuring an uncommon large bright still area in addition to the common dim propagating bullet area. Moreover, one of these plumes also contains a highly-conducting dark channel behind the bright area. Importantly, these highly-unusual plasma plumes were generated using short interruptions of a continuous high-voltage DC supply, which is also a simpler and energy-efficient alternative to the commonly used short DC pulses. The observed effects are explained using 
nanosecond time-resolved imaging of the discharge dynamics over the very short voltage rise and fall phases. This deterministic approach offers a simple and versatile control of the jet channel structure and conductivity as well as the plasma bullet propagation vitally needed for next-generation applications.
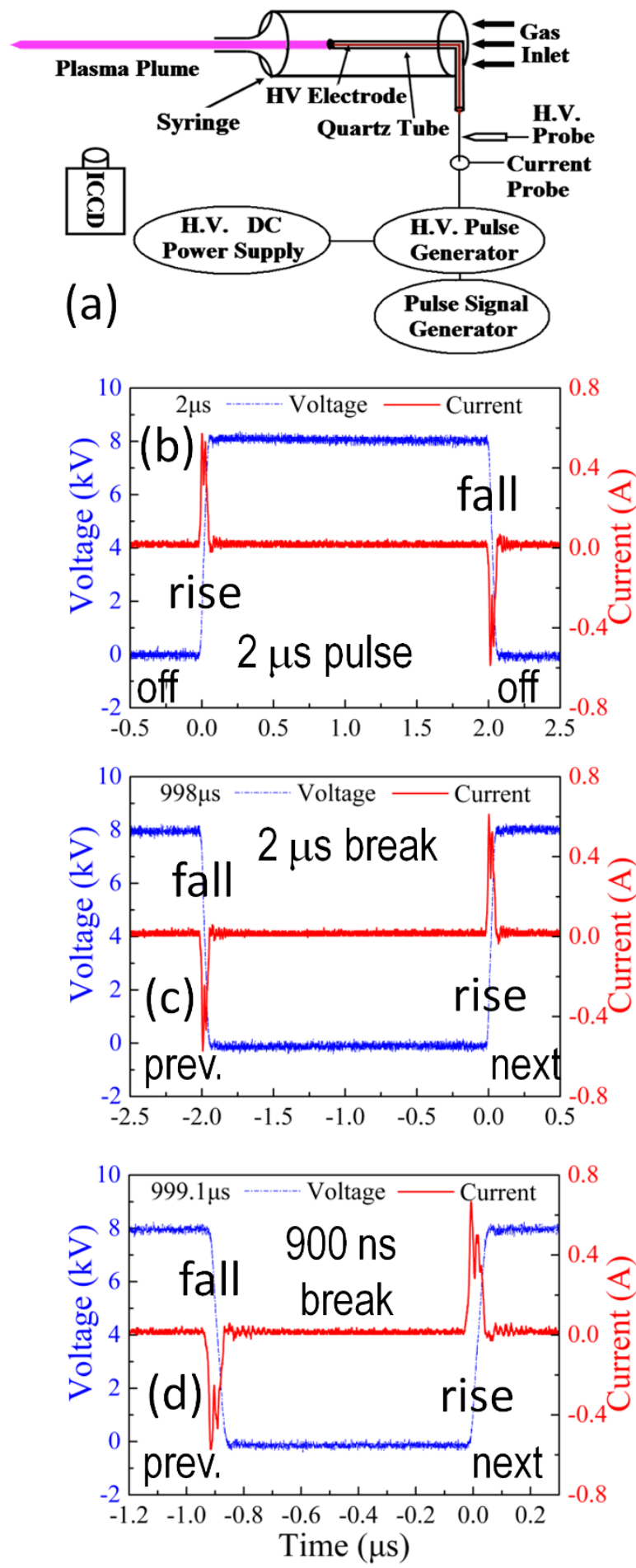

Figure $1 \mid$ Atmospheric plasma jet and DC pulse waveforms.

(a) Schematic of the atmospheric plasma jet device, gas feed, power supply, and plasma diagnostics. Current-voltage pulsed DC waveforms corresponding to the pulse width (b) $2 \mu \mathrm{s}$, one single pulse is shown; (c) $998 \mu \mathrm{s}$, a $2 \mu \mathrm{s}$ interruption between two pulses is shown; and (d) $999.1 \mu \mathrm{s}$, a $900 \mathrm{~ns}$ interruption between two pulses is shown. Breakdown occurs only during the voltage rise and fall phases.

\section{Results}

To demonstrate the highly-unusual plasma bullet-prone jet plumes by controlling the residual electrons, we used the atmospheric plasma jet device sketched in Fig. 1(a). The voltage waveforms used are shown in Figs. 1(b)-(d). These waveforms were chosen to: (i) produce the breakdown merely during the rise and fall phases of the voltage pulse; and (ii) trigger the next voltage rise or fall phase with the delay comparable with the lifetime of the residual electrons produced during the previous discharge phase. Using a typical observable plasma bullet propagation time of $1 \mu$ s as an (assumed) estimate of the residual electrons lifetime, the duration of such a delay was varied from $900 \mathrm{~ns}$ to $2 \mu \mathrm{s}$.

Fig. 1(b) shows a single $8 \mathrm{kV}$ DC voltage pulse of a $2 \mu$ s duration (and $1 \mathrm{~ms}$ repetition rate) and the current it produces during the initial rise phase and the following fall phase. The continuous $8 \mathrm{kV}$ DC voltage was also repeatedly interrupted for $2 \mu \mathrm{s}$, also every $1 \mathrm{~ms}$, as shown in Fig. 1(c). The interruption time was also reduced to 900 ns retaining the same repetition rate (Fig. 1(d)). During the interrupted DC operation, the fall phase preceded the rise phase as shown in Fig. 1(c,d). In all cases, the discharges were only produced during the rise or fall phases as evidenced by the current spikes in Fig. 1(b-d). This was also chosen deliberately to clearly separate the different discharge phases during the high-speed, time-resolved imaging and focus on the plasma bullet propagation which was previously reported to merely occur during the rise phase ${ }^{13}$.

Remarkably, all the three waveforms in Fig. 1(b-d) produced three totally different plasma plumes depicted in Fig. 2(a-c). The $2 \mu \mathrm{s}$ pulse has generated a common plume with a large dim area indicative of a propagating bullet (Fig. 2(a)). Importantly, this type of the plasma plumes was generated using DC pulses with the duration up to $990 \mu \mathrm{s}$ at the same repetition rate of $1 \mathrm{~ms}$. However, the other two interrupted DC waveforms produced exotic plumes with two and three distinctive areas labelled in Fig. 2(b) and (c), respectively. When the break between the pulses was $2 \mu$ s (Fig. 2(b)), the plume appears as a bright channel (area I) and a dim (likely bullet propagation) area II. This type of structures was revealed for the $2-10 \mu \mathrm{s}$ interruptions of $8 \mathrm{kV}$ DC pulses.

When the duration of DC interruption was reduced to less than $1 \mu$ s, a very different plume structure was discovered (Fig. 2(c)). One can clearly see the dark area I, the bright area II, and the dim (likely bullet propagation) area III in the plume generated using a $900 \mathrm{~ns}$ interruption (Fig. 1(d)).

To help explaining the observed exotic plume structures, timeresolved, high-speed imaging was implemented selectively during the rise and fall phases of each of the waveforms in Fig. 1. In all cases, it was found that the plasma bullets are only produced during the rise

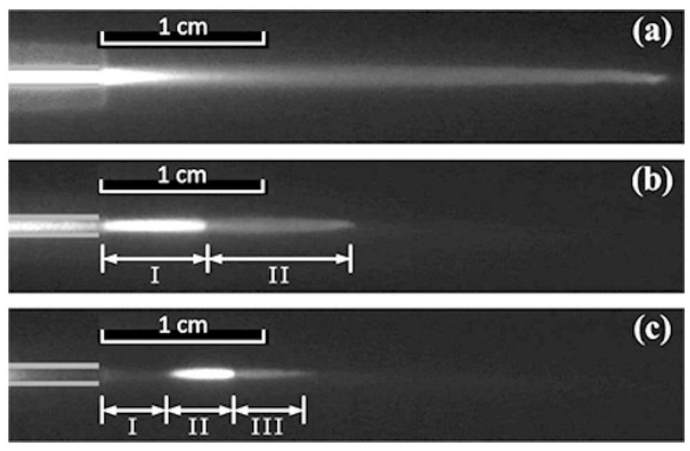

Figure $2 \mid$ Photographs of three distinctive plasma plume types generated using. (a) $2 \mu$ s pulse; mostly a dim (typical to a plasma bullet) area is seen; (b) $2 \mu$ s interruption; bright (I) and $\operatorname{dim}$ (II) areas are seen; and (c) $900 \mathrm{~ns}$ interruption; dark (I), bright (II), and $\operatorname{dim}$ (III) areas are seen. Images (a)(c) produced using a $1 \mu \mathrm{s}$ exposure from the beginning of the voltage rise phases labelled " 0 " in Figs. 1(b)-1(d), respectively. 
phases, for both the short pulses and the short interruptions. These high-speed images for the $2 \mu$ s pulse and the $2 \mu$ s and 900 ns breaks are shown in Figs. 3-5, respectively. The beginning of the time count was synchronized with the onset of the voltage rise phase. From these images one can clearly spot the very different discharge dynamics in each case.

When a short $2 \mu$ s pulse is used, the plume dynamics (Fig. 3) is typical to the plasma bullet propagation reported previously ${ }^{13}$. The bright plasma channel originates within the nozzle and starts exiting it at $528 \mathrm{~ns}$ into the pulse. At $628 \mathrm{~ns}$, the plume elongates to $\sim 1 \mathrm{~cm}$ forming a bright bullet at its head. $100 \mathrm{~ns}$ later, the bullet separates from the rest of the plume. Other images in Fig. 3 show that the bullet propagates further, with the speed of the ionization front $\sim 10^{5} \mathrm{~m} / \mathrm{s}$ (note that the speed of the gas flow is much lower, typically of the order of $\sim 10 \mathrm{~m} / \mathrm{s}$ ), eventually slowing down and shrinking in size. The rest of the plasma plume darkens significantly while the bullet propagates.

Fig. 4 shows a very different dynamics of the plasma plume generated by a short $2 \mu$ s interruption. The plume brightens and exits the nozzle much faster thereby forming a bright, non-propagating channel at 278 ns which then fully exits from the nozzle 100 ns later. The length of this channel is approximately $6 \mathrm{~mm}$. At $428 \mathrm{~ns}$, the channel brightens and the plasma bullet forms and tends to detach from the channel. $50 \mathrm{~ns}$ later, the bullet is fully detached and a dark channel forms between the head of the bright plasma channel and the small bullet-like plasma. As the bullet propagates further, its speed and size decrease while the luminosity of the bright channel I in Fig. 2(b) decreases. The speed and the length of the bullet propagation are noticeably smaller than in Fig. 3 thereby leading to a shorter plasma plume in Fig. 2(b) compared to Fig. 2(a). For example, the propagation speed of the bullet is approximately 3 times smaller than in the $2 \mu$ s pulse case.

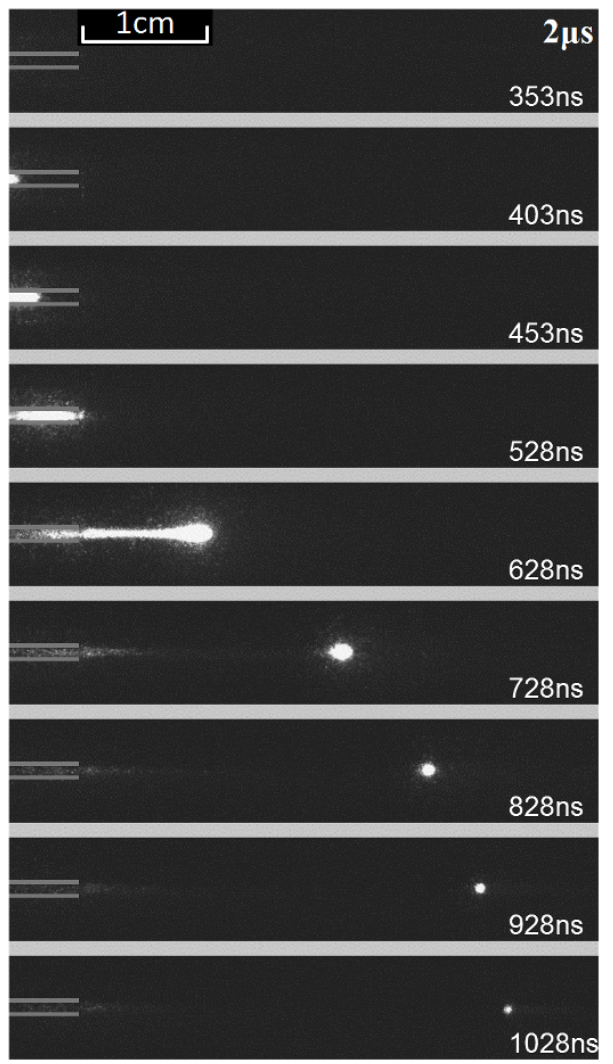

Figure $3 \mid$ High-speed photographs of the plasma plume generated during the voltage rise phase of the $2 \mu$ s pulse. The exposure time is 5 ns. The time labeled on each photograph corresponds to the time in Fig. 1(b).

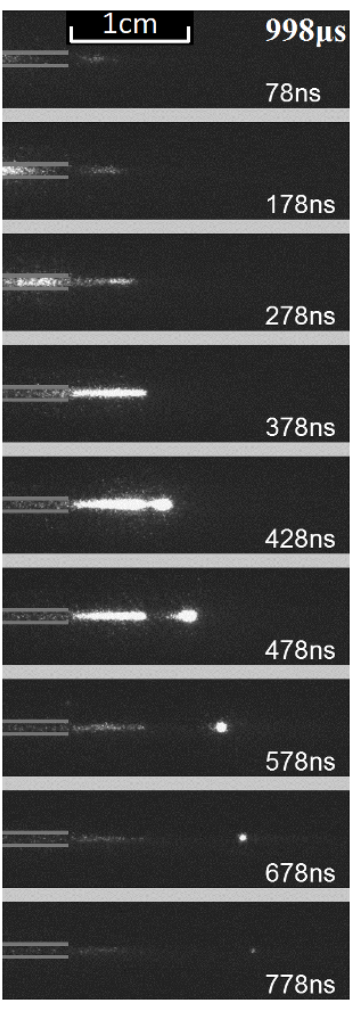

Figure $4 \mid$ Same as in Fig. 3 for the $2 \mu$ s DC interruption. The time labeled on each photograph corresponds to the time in Fig. 1(c).

In order to elucidate whether the bright plasma channel I in Fig. 2(b) is formed locally or is due to the integrated effect of a fast moving bullet, a dedicated experiment using the ICCD camera with an exposure time of 2 ns and a step of 2 ns was performed. This highspeed imaging confirms that the bright plasma channel at $378 \mathrm{~ns}$ is clearly not due to the integrated effect of a fast moving bullet.

If the break between the voltage fall and rise phases is further reduced, the bright plasma channel starts forming within the nozzle and then moves forward much faster. This can be seen in Fig. 5 which corresponds to the waveform in Fig. 1(d). Already at 173 ns, the bright channel clearly separates from the nozzle and leaves an

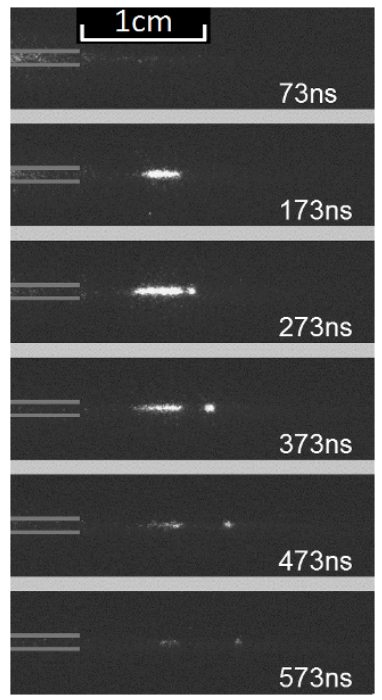

Figure $5 \mid$ Same as in Fig. 3 for the 900 ns DC interruption. The time labeled on each photograph corresponds to the time in Fig. 1(d). 
approx. $4 \mathrm{~mm}$ dark channel behind. The length of the dark channel between the nozzle and the left end of the bright plasma channel becomes smaller when the voltage interruption time decreases. At about $273 \mathrm{~ns}$, a plasma bullet is formed at the right end of the bright plasma channel and starts propagating forward. $100 \mathrm{~ns}$ later, a clear gap between the bullet and the channel is visible. At this moment, the channel becomes dimmer and this tendency continues thereafter. Interestingly, the length of the dark space (area I in Fig. 2(c)) between the nozzle and the left end of the bright channel does not noticeably change with time. As the bullet propagates, it also reduces in size and slows down, similar to the two other cases. The propagation speed of the bullet in Fig. 5 is somewhat smaller than in Fig. 4.

\section{Discussion}

Here we discuss the observed phenomena and relate them with the density and response of residual electrons, in the context of the existing knowledge on the propagation mechanism of pulsed atmospheric plasma streamers. We will then summarize the interpretations of the key observations and the remaining open issues.

Dark channel. Numerical modelling shows that electron density of dark channel left behind the plasma bullet is typically of the order of $10^{11}-10^{12} \mathrm{~cm}^{-3}{ }^{17}$. The high electron density results in a high conductivity of the dark channel. Thus, the electric field within the dark channel is low, typically about a few $\mathrm{kV} / \mathrm{cm}^{17}$. Hence, the dark channel effectively serves as an extended electrode. This is also the reason why the plasma bullet propagation is inhibited at the falling edge of the applied voltage $\mathrm{e}^{18}$. Because the conductivity of the dark channel is high, the plasma bullet is electrically connected to the high-voltage electrode.

The electron density in the dark channel in Fig. 3 decreases with time and distance from the nozzle. The electron density in the dark channel behind the plasma bullet in Fig. 5 is also expected to behave quite similarly because of the similar trends of the electron density decay, as in the case of the pulse width of $2 \mu$ s. Quantitatively, the electron densities in the two dark channels in Fig. 5 are quite different over the most of the pulse cycle. The dark channel behind the bright channel has a lower mole fraction of air than the dark channel behind the plasma bullet. Consequently, the electron density behind the bright channel experiences a slower decay than behind the plasma bullet. More importantly, the electrons in these two dark channels are generated at different time. While the electrons in the dark channel behind the bright channel are generated during the discharge at both the falling and the rising edges of the pulse, the electrons in the dark channel behind the plasma bullet are generated during the discharge at the rising edge of the pulse (the discharge at the falling edge does not reach this position as can be seen in Fig. S3). It is estimated that the electron density before the falling edge discharge in the dark channel behind the bright channel is of the order of $10^{9} \mathrm{~cm}^{-3}$, while in the dark channel behind the plasma bullet it is of the order of $10^{4} \mathrm{~cm}^{-3}$. This difference is due to the different $\mathrm{O}_{2}$ concentration. After the falling edge discharge, the electron density in the dark channel behind the bright channel rise to $\sim 10^{12} \mathrm{~cm}^{-3}$, while the electron density in the dark channel behind the plasma bullet remains much lower (of the order of $10^{4} \mathrm{~cm}^{-3}$ ) because the plasma did not reach the position. For the pulse width of $999.1 \mu$ s, the discharge at the rising edge is ignited only several hundred ns after the discharge at the previous falling edge. Then the electron density in the both dark channels could reach the value of the order of $\sim 10^{12} \mathrm{~cm}^{-3}$.

2-10 $\mu$ s breaks between voltage phases. In our experiments, when the voltage interruptions are longer than $2 \mu \mathrm{s}$ but shorter than $10 \mu \mathrm{s}$, a bright channel is generated in front of the nozzle at the rising edge. A plasma bullet is generated at the right tip of the bright channel and moves forward. This phenomenon does not appear when the voltage break is longer than $10 \mu \mathrm{s}$. It is thus reasonable to assume that the observed unusual plasma plume structure is due to the increase of the residual electron density left from the discharge at the falling edge of the previous voltage pulse. When the time gap between the rise phase and the preceding fall phase becomes shorter so does the break between the two consecutive discharges ignited during the both phases. In this case, species produced by the fall-phase discharge may not completely recombine before the onset of the subsequent discharge at the voltage rise phase. Therefore, because of the short time between the two discharges, the density of residual electrons produced during the preceding fall phase may remain high before the next discharge is ignited at the rising edge of the voltage pulse. We emphasize that the length of the plasma plume during the fall phase is much shorter compared to the rise phase, as can be clearly seen from Fig. 4 and Fig. S3(b). From Fig. 2(b) one can also note that the length of the bright section I is close to the length of the plasma channel deduced from high-speed imaging in Supplementary Fig. S3. It is thus reasonable to assume that the residual electron density within section I is relatively high.

Consequently, section I becomes conductive at the onset of the rise phase. The dark channel is left from the propagation of the plasma bullet. The reason why section I appears bright is probably because the electric field within section I is higher than within the dark channel. The relatively high electric field within section I sustains a certain degree of ionization and excitation, which is balanced by the recombination, attachment and diffusion of the gas species. This is why the plasma of section I remains stable for a relatively long time as evidenced in Fig. 4. For section II shown in Fig. 2(b), the residual electron density is much lower than in section I which helps sustaining the plasma bullet propagation as seen in Fig. 4. The numerical modelling of the distribution of the residual electron density is presented in Sec. S4 of Supplementary Information. As shown in Fig. S7, when the pulse width is $998 \mu$ s, the residual electron density is about $4 \times 10^{11} \mathrm{~cm}^{-3}$ in section I in Fig. 2 (b) and about $10^{4} \mathrm{~cm}^{-3}$ in section II in Fig. 2(b). This result is consistent with the phenomenological discussions in this work.

Shorter $(<2 \mu \mathrm{s})$ breaks between voltage phases. When the voltage interruption is shorter than $2 \mu \mathrm{s}$, the residual electron density in front of the nozzle is even higher when the discharge is ignited during the rise phase. Hence, section I in Fig. 2(c) has an even higher electric conductivity and a weaker electric field. The weaker electric field results in a low electron temperature, so the electrons cannot gain enough energy to ionize or excite atoms or molecules.

Role of air diffusion. It should be pointed out that the diffusion of air plays a significant role in the phenomena discussed above. Due to the diffusion, the air concentration becomes higher and higher in the $\mathrm{He}$ stream with the increase of the distance from the nozzle. Because of the very high electron affinity of oxygen, the high concentration of air in the He flow results in the fast decay of the residual electron density. This is why the residual electron density decreases away from the nozzle. Consequently, sections I and II in Fig. 2(c) feature the high and medium residual electron density. This in turn results in the dark region I, and bright region II, respectively. As shown in Figure S7, when the pulse width is $999.1 \mu \mathrm{s}$, the residual electron density decreases with the distance from the nozzle in sections I and II in Fig. 2(c) (see Sec. S4 in Supplementary Information). This numerical result further supports our discussion.

Moreover, diffusion of oxygen and nitrogen species contributes to de-excitation of He metastables, which contributes to the optical emission, see the optical emission spectra in Figure S5 in Supplementary Information. The mechanism is related to the Penning ionization whereby these molecules are ionized. It is well known that Penning ionization can enhance the discharge. It increases the electron density and the velocity of the plasma bullet. This is why this effect may be considered as counteracting to the effect of the decrease 
of the residual electron density. The resulting optical emission from the plasma plume is a consequence of the ionization-excitation-radiation balance. The residual electron- and helium metastable-related mechanisms both contribute to this balance, and consequently, to the observed discharge behaviour.

Summary of observations and interpretations. We have thus observed two new phenomena produced when the duration of interruption of DC voltage are comparable with the lifetime of the residual electron density. First, when the break is between $2 \mu \mathrm{s}$ and $10 \mu \mathrm{s}$, the plasma jet generated during the voltage rise phase can be divided into two sections. In section I which appears next to the nozzle, the plasma appears like a bright channel and has a length of several millimetres. The bright channel appears in section I and a plasma bullet is generated at the tip of the bright channel. The bullet then detaches and propagates along the He stream. The bright channel is due to the high residual electron density left from the discharge at the preceding voltage fall phase. The high residual electron density results in the relatively high electric conductivity and hence a weak electric field. The electron density of the bright channel of section I is lower compared to the dark channel due to recombination, attachment, and other loss effects. Consequently, the electric field within section I is stronger than in the dark channel. This electric field leads to the higher electron temperature which in turn raises the level of excitation of atomic/molecular species in the gas flow. This produces intense optical emission from section I. For section II in Fig. 2(b), the residual electron density is much lower compared to section I and the plasma retains the propagating bullet behaviour.

Second, when the voltage break is further reduced, the gap between the discharge at the rise phase and the discharge at the preceding fall phase becomes shorter and shorter, which increases the residual electron density in the channel. The bright plasma channel (section II in Fig. 2(c)) detaches further and further away from the nozzle as the voltage break decreases. This creates a dark channel (section I in Fig. 2(c)) between the nozzle and the left end of the bright plasma channel. On the right end of the bright plasma channel, the jet still retains the plasma bullet behaviour. Due to the air diffusion into the He stream and the discharge at the falling edge of the previous voltage pulse only reaching sections I and II, the residual electron density in sections I, II, and III of Fig. 2(c) is high, medium, and low, respectively and the residual electron density in sections I and II are many orders higher than that in section III. This difference in the electron densities results in the appearance of the dark, bright, and bullet areas, respectively. The numerical modelling of the distribution of the residual electron density presented in Sec. S4 of Supplementary Information supports this explanation.

Therefore, our results suggest that the residual electron density in the discharge channels of atmospheric-pressure plasma jets plays a critical role in the formation, structure, and temporal dynamics of the plasma plumes. This phenomenon has not been studied previously despite a large number of studies on the effect of seed electrons on the streamer propagation. Precise tailoring of the duration and ramping of DC power supply appears to be a viable way to achieve the exotic multi-segmented plumes and control the plasma bullet formation and propagation. Interaction of the plasma jet with the surrounding air is also a decisive factor in the spatial distribution and dynamics of the residual electron density. Detailed study of this interaction and other possible mechanisms is therefore highly warranted in the near future. For example, the absorption of photoemission by $\mathrm{He}, \mathrm{N}_{2}$ and $\mathrm{O}_{2}$ may also play a significant role in the streamer propagation ${ }^{31}$.

Potential applications. The demonstrated control of the plasma bullet formation and propagation is highly-promising for the controlled delivery of quantized bits of plasmas with micrometer and nanosecond precision, which may open new exciting prospects for applications in cellular-level medical therapies and selective treatment of microscopic objects in numerous materials and device technologies. Indeed, although the typical size of the spot of the plasma jet is in the sub-mm range, it can be reduced to a couple of hundreds or even a few tens of micrometers by a proper optimization of the discharge parameters. Apart from the small dimensions of the localized treatment spots, the surface temperature within these spots can reasonably be expected to be close to room temperature. This is particularly important for the synthesis and processing of temperature-sensitive materials such as polymers ${ }^{32,33}$. Moreover, low energies of the plasma ions (the ion velocities in front of the plasma bullet are of the order of $\sim 10^{3} \mathrm{~m} / \mathrm{s}$, which is much lower than typically in low-pressure plasmas) are very favourable for the treatment of materials that are highly-susceptible to ion damage such as delicate graphenes and single-walled carbon nanotubes ${ }^{34,35}$. These interesting features of the pulsed atmospheric pressure streamers may bring several advantages to the field of nanoscale synthesis and processing where low-pressure plasmas have demonstrated a number of advantages over many other conventional methods ${ }^{36-38}$.

\section{Methods}

Fig. 1(a) is the schematic of the experimental setup. The plasma plume is generated by a single electrode plasma jet device. The voltage pulse repetition rate $(\mathrm{ON}+\mathrm{OFF}$ pulse sequences) is fixed at $1 \mathrm{kHz}$ throughout this paper. The pulse width was varied from $900 \mathrm{~ns}$ to $999.1 \mu \mathrm{s}$. The applied voltage is measured by a P6015A Tektronix HV probe and the current by a Pearson 6585 current probe. A fast ICCD camera (Princeton Instruments, Model: PIMAX2) is used to capture the nanosecond dynamics of the discharge. The exposure time is fixed at $5 \mathrm{~ns}$ for the photographs shown in this paper. A $1 \mu$ s exposure is used to produce still images of the plasma plumes in Fig. 2. The terms "dim", "dark", and "bright" are used to describe the relative luminance in different areas of the plasma jet rather than to express their absolute luminance. Similar presentations of the relative luminance such as "dark space", "glow" are consistently used to describe the visual appearance of the glow discharge. The discharge currents shown in Fig. 1 are the total currents and represent the sum of the displacement current and the actual discharge current, where the actual discharge current is much smaller than the displacement current. It should be mentioned that the gas temperature may be affected by the pulse repetition frequency; however, this frequency was fixed at $1 \mathrm{kHz}$ in our experiments. We have also observed that when varying the pulse width, the discharge current also changes; however, this change is fairly small. As a result, the gas temperature does not undergo any significant changes. During the jet operation at a fixed pulse repetition frequency, the gas temperature remains close to room temperature. This is why the assumption of the constant near-room temperature is reasonable. For each waveform in Fig. 1, the zero time moment is set at the beginning of the rise phase. The reason for this choice was to temporally resolve the plasma bullet propagation which was only observed during the voltage rise phase. The gas flow used in this series of experiments was 1 $1 / \mathrm{min}$. Under such conditions, the gas flow is laminar and the pulsed atmospheric pressure streamer propagates in a linear fashion. Above $\sim 5 \mathrm{l} / \mathrm{min}$, turbulent gas flows develop, which make the streamer propagation more sophisticated. For example, it was observed that the plasma bullet may exhibit a snake-like propagation, which was intentionally avoided for the purpose of this work. More details about the plasma jet device, the associated measurements and the method used to calculate the electron density can be found in Supplementary Information.

1. Laroussi, M. Low-Temperature Plasmas for Medicine? IEEE Trans. Plasma Sci. 37(6), 714-725 (2009).

2. Schubert, G., Basner, R. \& Kersten, H. Determination of sheath parameters by test particles upon local electrode bias and plasma switching. Eur. Phys. J. D 63(3), 431-440 (2011)

3. Kong, M., Kroesen, G., Morfill, G., Nosenko, T., Shimizu, T., Dijk, J. \& Zimmermann, J. Plasma medicine: an introductory review. New J. Phys. 11, 115012 (2009).

4. Fridman, G. et al. Applied plasma medicine. Plasma Process. Polym. 5(6), 503-533 (2008).

5. Morfill, G. E., Kong, M. G. \& Zimmermann, J. L. Focus on Plasma Medicine. New J. Phys. 11, 115011 (2009)

6. Ostrikov, K., Cvelbar, U. \& Murphy, A. B. Plasma nanoscience: setting directions, tackling grand challenges. J. Phys. D: Appl. Phys. 44 (17), 174001 (2011).

7. Mariotti, D. \& Sankaran, R. M. Microplasmas for nanomaterials synthesis. J. Phys. D: Appl. Phys. 43 (32), 323001 (2010).

8. Isbary, G. et al. Successful and safe use of 2 min cold atmospheric argon plasma in chronic wounds: results of a randomized controlled trial. British J. Dermatology 167(2), 404-410 (2012).

9. Maurer, H. R. \& Kersten, H. On the heating of nano- and microparticles in process plasmas. J. Phys. D: Appl. Phys. 44(17), 174029 (2011). 
10. Volotskova, O., Stepp, M. A. \& Keidar, M. Integrin activation by a cold atmospheric plasma jet. New J. Phys. 14, 053019 (2012).

11. Chen, W. et al. Treatment of enterococcus faecalis bacteria by a helium atmospheric cold plasma brush with oxygen addition. J.Appl. Phys. 112(1), 013304 (2012)

12. Teschke, M., Kedzierski, J., Finantu-Dinu, E. G., Korzec, D. \& Engemann, J. Highspeed photographs of a dielectric barrier atmospheric pressure plasma jet. IEEE Trans. Plasma Sci. 33(2), 310-311 (2005).

13. Lu, X. \& Laroussi, M. Dynamics of an atmospheric pressure plasma plume generated by submicrosecond voltage pulses. J. Appl. Phys. 100(6), 063302 (2006).

14. Ono, R., Nakagawa, Y. \& Oda, T. Effect of pulse width on the production of radicals and excited species in a pulsed positive corona discharge. J. Phys. D: Appl. Phys. 44(48), 485201 (2011).

15. Bibinov, N., Knake, N., Bahre, H., Awakowicz, P. \& Schulz-von der Gathen, V. Spectroscopic characterization of an atmospheric pressure mu-jet plasma source. J. Phys. D: Appl. Phys. 44(34), 345204 (2011).

16. Clevis, T. T. J., Nijdam, S. \& Ebert, U. Inception and propagation of positive streamers in high-purity nitrogen: effects of the voltage rise-rate. J. Phys. D: Appl. Phys. 46(4), 045202 (2013).

17. Naidis, G. V. Modelling of streamer propagation in atmospheric-pressure helium plasma jets. J. Phys. D: Appl. Phys. 43(40), 402001 (2010).

18. Mericam-Bourdet, N., Laroussi, M., Begum, A. \& Karakas, E. Experimental investigations of plasma bullets. J. Phys. D: Appl. Phys. 42(5), 055207 (2009).

19. Breden, D., Miki, K. \& Raja, L. L. Self-consistent two-dimensional modeling of cold atmospheric-pressure plasma jet/bullets. Plasma Sources Sci. Technol. 21(3), 034011 (2012).

20. Xiong, Z. \& Kushner, M. J. Atmospheric pressure ionization waves propagating through a flexible high aspect ratio capillary channel and impinging upon a target. Plasma Sources Sci. Technol. 21(3), 034001 (2012).

21. Sakiyama, Y., Graves, D. B., Jarrige, J. \& Laroussi, M. Finite element analysis of ring-shaped emission profile in plasma bullet. Appl. Phys. Lett. 96(4), 041501 (2010).

22. Leiweke, R. J., Sands, B. L. \& Ganguly, B. N. Effect of Gas Mixture on Plasma Jet Discharge Morphology. IEEE Trans. Plasma Sci. 39(11), 2304-2305 (2011).

23. Kolb, J. et al. Cold atmospheric pressure air plasma jet for medical applications. Appl. Phys. Lett. 92(24), 241501 (2008).

24. Yousfi, M., Eichwald, O., Merbahi, N. \& Jomaa, N. Analysis of ionization wave dynamics in low-temperature plasma jets from fluid modeling supported by experimental investigations. Plasma Sources Sci. Technol. 21(4), 045003 (2012).

25. Wormeester, G., Pancheshnyi, S., Luque, A., Nijdam, S. \& Ebert, U. Probing photo-ionization: simulations of positive streamers in varying $\mathrm{N} 2$ :O2-mixtures. J. Phys. D: Appl. Phys. 43(50), 505201 (2010).

26. Tholin, F. \& Bourdon, A. Influence of temperature on the glow regime of a discharge in air at atmospheric pressure between two point electrodes. J. Phys. D: Appl. Phys. 44(38), 385203(2011).

27. Liu, F. C., Zhang, D. Z. \& Wang, D. Z. The influence of air on streamer propagation in atmospheric pressure cold plasma jets. Thin Solid Films, 521, 261-264 (2012).

28. Komuro, A., Ono, R. \& Oda, T. Numerical simulation for production of $\mathrm{O}$ and $\mathrm{N}$ radicals in an atmospheric-pressure streamer discharge. J. Phys. D: Appl. Phys. 45(26), 265201 (2012).

29. Naidis, G. V. Modelling of plasma bullet propagation along a helium jet in ambient air. J. Phys. D: Appl. Phys. 44(21), 215203 (2011).
30. Boeuf, J. P., Yang, L. L. \& Pitchford, L. C. Dynamics of a guided streamer ('plasma bullet') in a helium jet in air at atmospheric pressure. J. Phys. D: Appl. Phys. 46(1), 015201 (2013).

31. Xiong, Z. M. \& Kushner, M. J. Atmospheric pressure ionization waves propagating through a flexible high aspect ratio capillary channel and impinging upon a target. Plasma Sources Sci. Technol. 21(3), 034001 (2012).

32. Vasilev, K., Griesser, S. \& Griesser, H. J. Antibacterial surfaces and coatings produced by plasma techniques. Plasma Proc. Polym. 8(11), 1010-1023 (2011).

33. Cvelbar, U., Junkar, I. \& Modic, M. Hemocompatible poly(ethylene terephthalate) polymer modified via reactive plasma treatment. Jpn. J. Appl. Phys. Part 2 50(8), 08JF02 (2011).

34. Lim, Y.-D. et al. Si-compatible cleaning process for graphene using low-density inductively coupled plasma. ACS Nano 6, 4410-4417 (2012).

35. Ostrikov, K. \& Mehdipour, H. Thin single-walled carbon nanotubes with narrow chirality distribution: constructive interplay of plasma and Gibbs-Thomson effects. ACS Nano 5, 8372-8382 (2011).

36. Xu, S., Levchenko, I., Huang, S. Y. \& Ostrikov, K. Self-organized vertically aligned single-crystal silicon nanostructures with controlled shape and aspect ratio by reactive plasma etching. Appl. Phys. Lett. 95, 111505 (2009).

37. Meyyappan, M. Plasma nanotechnology: past, present and future. J. Phys. D: Appl. Phys. 44(17), 174002 (2011)

38. Ostrikov, K., Xu, S. \& Levchenko, I. Self-organized nanoarrays: plasma-related controls. Pure Appl. Chem. 80, 1909-1918 (2008).

\section{Acknowledgements}

This work was partially supported by the National Natural Science Foundation (Grant No. $51077063,51277087)$, Research Fund for the Doctoral Program of Higher Education of China (20100142110005), and the Chang Jiang Scholars Program, Ministry of Education, People's Republic of China, the Australian Research Council and CSIRO's CEO Science Leadership Program.

\section{Author contributions}

X.L. led the project and supervised all experiments. Y.X., P.Z., X.P., S.W. and Q.X. conducted experiments and measurements. X.L. and K.O. co-led data analysis and physical interpretations. All authors discussed the results. Y.X., X.L. and K.O. co-wrote the manuscript.

\section{Additional information}

Supplementary information accompanies this paper at http://www.nature.com/ scientificreports

Competing financial interests: The authors declare no competing financial interests.

License: This work is licensed under a Creative Commons

Attribution-NonCommercial-NoDerivs 3.0 Unported License. To view a copy of this license, visit http://creativecommons.org/licenses/by-nc-nd/3.0/

How to cite this article: Xian, Y. et al. From short pulses to short breaks: exotic plasma bullets via residual electron control. Sci. Rep. 3, 1599; DOI:10.1038/srep01599 (2013). 